Research Article

\title{
Urban Traffic Signal Control Based on Multiobjective Joint Optimization
}

\author{
Yongrong Wu, ${ }^{1}$ Yijie Zhou, ${ }^{1}$ Yanming Feng, ${ }^{1}$ Yutian Xiao, ${ }^{2}$ Shaojie He, ${ }^{2}$ Junsheng Zhou, ${ }^{2}$ \\ Tianhe Ren, ${ }^{2}$ Jinling Chen, ${ }^{2}$ Mingsong Chen $\left(\mathbb{b},{ }^{1}\right.$ Jianbing Xiahou $\left(\mathbb{D},{ }^{2}\right.$ and Fan Lin $\left(\mathbb{D}^{2}\right.$ \\ ${ }^{1}$ School of Information and Communication, Guilin University of Electronics Technology, Guilin 541004, China \\ ${ }^{2}$ Xiamen University, Xiamen 361001, China \\ Correspondence should be addressed to Mingsong Chen; cms@guet.edu.cn, Jianbing Xiahou; jbxiahou@xmu.edu.cn, and \\ Fan Lin; iamafan@xmu.edu.cn
}

Received 7 April 2020; Revised 25 May 2020; Accepted 10 August 2020; Published 15 October 2020

Academic Editor: Chao Huang

Copyright $(92020$ Yongrong Wu et al. This is an open access article distributed under the Creative Commons Attribution License, which permits unrestricted use, distribution, and reproduction in any medium, provided the original work is properly cited.

This paper proposes two algorithms for signal timing optimization of single intersections, namely, microbial genetic algorithm and simulated annealing algorithm. The basis of the optimization of these two algorithms is the original timing scheme of the SCATS, and the optimized parameters are the average delay of vehicles and the capacity. Experiments verify that these two algorithms are, respectively, improved by $67.47 \%$ and $46.88 \%$, based on the original timing scheme.

\section{Introduction}

In order to solve the current traffic problem more effectively, this paper proposes a single intersection signal control method. The traffic signal control of a single intersection is the smallest unit of the entire city traffic signal control system. It plays an important role in improving traffic order at intersections, reducing vehicle delays, and improving traffic capacity [1]. With the continuous deepening of the concept of sustainable development of urban transportation, the goal of traffic signal control is gradually changing from a single goal to multiple goals. Therefore, the multiobjective joint optimization of traffic signal control has attracted researchers' attention.

When optimizing traffic signal parameters, many factors need to be considered comprehensively, such as average delay, number of stops, and queue length [2]. Among them, the most commonly used criteria are delay and number of stops. These service quality measures are considered to be the most important factors for users to perceive the service level [3]. Webster established a steady-state random delay model for unsaturated traffic flow [4]. Heydecker and Wey used the average delay of motor vehicle flow at the intersection as the optimization target of signal control parameters [5, 6]. Robertson used a linear combination of optimization goals such as average delay and number of stops to establish an optimized model of signal control parameters $[7,8]$.

The multiobjective problem of traffic control has become a hotspot in current research, which has aroused extensive attention of researchers. Zeng et al. established a multiobjective optimization model of signal control parameters. The cell transmission model was used to simulate the operation law of motor vehicle flow at signal control intersections. The average delay and capacity were used as optimization goals [9]. Taking the average delay, average parking rate, and capacity as the optimization goals, Yao et al. established a multiobjective optimization model but did not give a specific solution algorithm [10]. Taking the average delay of motor vehicle flow, average parking rate, and pedestrian waiting time as optimization goals, $\mathrm{Ma}$ et al. established a multiobjective optimization model for the period duration of a single intersection with fixed period signal control [11]. Li and Lu took the average delay and average number of vehicle stops at intersections as the optimization goals, established a multiobjective optimization model of signal control parameters for a single 
intersection, and used genetic algorithms for optimization [12]. Taking the average delay of motor vehicle flow, the average number of stops, and the total passing traffic as the optimization goals, $\mathrm{Cao}$ and $\mathrm{Xu}$ established a weighted combination optimization model of signal control parameters for single intersection with saturation constraints and solved the model using genetic algorithm [13].

Although there are many models for multiobjective joint optimization, there are no studies comparing the actual vehicle flow at the intersection with the average delay of vehicles and the capacity of the intersection as variables, compared with the original SCATS. Therefore, the optimization algorithm in this paper combines the actual intersection road conditions to analyze the night peak traffic information, which is an innovative point.

\section{Signal Timing Method Based on Multiobjective Joint Optimization}

The basic idea of signal distribution is to allocate time resources to traffic lights at intersections according to the traffic flow in each direction, so as to conduct traffic guidance. It is very important for signal synchronization [14]. In general, the best period should be the average period. If the period is too long to significantly improve the traffic capacity, it will lead to further delays of vehicles [15]. According to the traffic distribution, geometry, intersection type, area, and relevant conditions of each intersection, each intersection has a corresponding optimal time period, so the optimal time period changes in different time periods. Traffic function and control intersection signals are the best cycle, which can provide the best length for the whole vehicle [16].

In real life, urban intersections often adopt the timing scheme, which is simple and easy to operate, but it has many limitations. Through the transformation of the Webster formula, we can get accurate signal timing for real-time traffic and improve the efficiencies of road. This paper analyzes the traffic flow of the same intersection at the same time through two algorithms and then compares these two algorithms with the classic timing scheme of SCATS.

2.1. Notes on SCATS. The SCATS [17], researched and developed by the New South Wales Road Traffic Authority (RTA), is one of the few advanced urban signal traffic control systems in the world. When the system is selected through a real-time solution, the real-time selection of the signal period and green ratio is based on overall demand.

The corresponding green light time of the intersection shall be determined in accordance with the principle that the saturation degrees of all phases are equal or close to each other [18]. With the adjustment of the signal period, the green time of each phase also changes.

2.2. Basis of Algorithm. This part will be introduced separately from the signal phase of the intersection, the model of the intersection, and the formula for calculating the objective functions.
2.2.1. Objective Function Calculation Formula. TRRL method is also known as Webster method or F-B method. This signal timing method and corresponding calculation formula were first proposed by Corbusier and Webster in the 1990s [19]. The basic idea of this model is to take the vehicle delay time as the only evaluation index when the vehicle passes the intersection and to optimize the signal timing scheme through calculation. According to the theory of signal timing at the intersection [20], the two decisive factors are signal period and green time.

The formula for calculating the objective function PI is as follows:

$$
\mathrm{PI}=\min \left(\alpha \frac{D}{D_{0}}-\beta \frac{B}{B_{0}}\right) .
$$

In (1), $D$ is the vehicle delay at the intersection, $B$ is the capacity of the intersection, and $\alpha$ and $\beta$ are two evaluation indicators, respectively, representing the proportion of vehicle delay and capacity (they are considered equally important in this paper, both assigned the value 0.5).

The formulas for calculating the vehicle delay $D$ and the capacity of the intersection $B$ are as follows:

$$
\begin{aligned}
& D=\frac{\sum D_{i} q_{i}}{\sum q_{i}} \\
& B=B_{A}+B_{B}+B_{C}+B_{D} .
\end{aligned}
$$

In (2) and (3), $D_{i}$ is the stop delay of each vehicle in phase $i, q_{i}$ is the flow in phase $i$, and $B_{i}$ is the capacity of the intersection in phase $i$.

The delay at the $i$ th phase intersection is $D_{i}$, and the traffic capacity at the $i$ th phase intersection is $B_{i}$. The calculation formulas for the two of them are as follows:

$$
\begin{aligned}
D_{i} & =\frac{C\left(1-\lambda_{i}\right)^{2}}{2\left(1-y_{i}\right)^{2}}+\frac{y_{i}^{2}}{2 q_{i} \lambda_{i}\left(\lambda_{i}-y_{i}\right)}, \\
B_{i} & =k_{i} \times \frac{3600}{h_{i}} \times \lambda_{i} .
\end{aligned}
$$

In (4) and (5), $C$ is the cycle, $\lambda_{i}$ is the green ratio in phase $i, y_{i}$ is the traffic intensity in phase $i, k_{i}$ is the number of lanes in phase $i$, and $h_{i}$ is the lane saturation headway in phase $i$ (the default value for this article is $2.5 \mathrm{~s}$ ).

The formula for period $C$ is as follows:

$$
C=\sum g_{i}+\sum\left(l_{i}+R_{i}\right)
$$

In (6), $g_{i}$ is the decision variable and there are 4 decision variables in total $\left(g_{A}, g_{B}, g_{C}, g_{D}\right), g_{A}$ is the effective green time of phase $A, g_{B}$ is the effective green time of phase $B, g_{C}$ is the effective green time of phase $C$, and $g_{D}$ is the effective green time of phase $D$.

That is to say, $C=g_{A}+g_{B}+g_{C}+g_{D}+$ (delay_start + delay_redlight) $\times 4$; in this paper, when the green light is on, delay-start is set to 3 seconds and delay-red light is set to 2 seconds. 
$\lambda_{i}$ is the green ratio in phase $i$, and the calculation formula is as follows:

$$
\lambda_{i}=\frac{g_{i}}{C} .
$$

In (7), $y_{i}$ is the traffic intensity in phase $i$, and the calculation formula is as follows:

$$
y_{i}=\frac{q_{n} h_{i}}{g_{i} k_{i}} .
$$

In (8), $q_{i}$ is the traffic flow in phase $i$.

The calculation of the average vehicle delay $D_{0}$ and the initial intersection capacity $B_{0}$ is similar to that of $D_{i}$ and $B_{i}$, but there is a difference in calculating the period and the green ratio; $C_{0}$ and $\lambda_{i 0}$ use the calculation method of timing period, and the calculation formula is as follows:

$$
\begin{aligned}
& C_{0}=\frac{1.5 L+5}{1-Y}, \\
& \lambda_{i 0}=\frac{\left(C_{0}-L\right) y_{i}}{C_{0} Y} .
\end{aligned}
$$

In (9) and (10), $L$ is the total delay time of four phases and $Y$ is the sum of the four phases of $y_{i}$.

The above formula is the basis of optimizing traffic signals in this paper. The following are a detailed introduction of the process of each algorithm and the final results from different algorithm perspectives.

2.2.2. Signal Phase and Intersection Model. Before determining which signal phase is used at the intersection, it is important to fully understand the condition of the vehicle as it rushes from all directions to the intersection.

In this paper, the investigation and research intersection is a three-lane intersection; after taking full account of the actual traffic situation, a four-phase traffic signal is set up. Based on the road near schools, supermarkets, residential areas, vegetable markets, and many other crowded and traffic-intensive areas, we set the signal allocation time separate for the turn (left or right), so as to alleviate the traffic problems that may face the turning vehicles, reducing the traffic pressure at the intersection. The four-phase traffic signal is shown in Figure 1, and the urban intersection model is shown in Figure 2.

The numbers 1-12 in Figure 2 are the coil numbers, which are used to calculate the ratio of the actual traffic volume at the intersection to the saturated traffic volume. The directions of the arrows nos. 1-4 are the same as the four phase directions A-D in Figure 1.

2.2.3. Experimental Data Source. The data source of this article is the real data of one hour $(17: 28-18: 30)$ of the night peak of a certain intersection. The set of data includes the effective green light duration of the four phases, the number of vehicles passing through the set green light cycle, and its coil number. Because of the large amount of recorded traffic

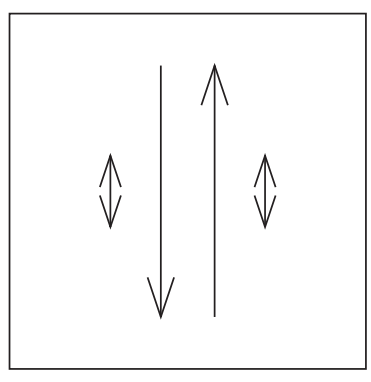

(a)

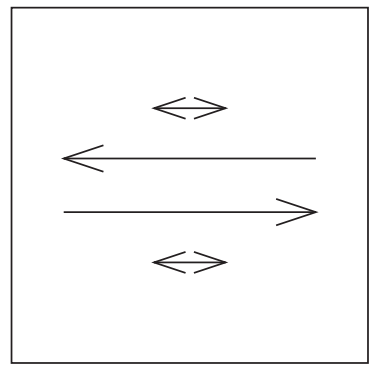

(c)

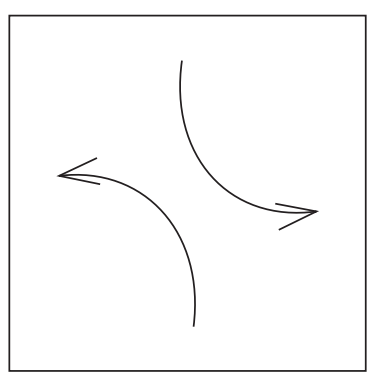

(b)

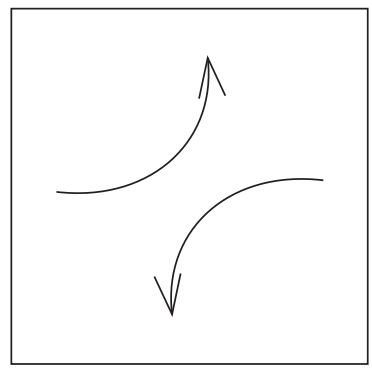

(d)
Figure 1: Phase of the traffic flow.

information, this article randomly selects 20 lists as templates.

In the comparative experiment of this article, there are four sets of timing schemes for the SCATS of the intersection of the test subject. Taking the third set of plans as reference, the green light ratio information of the four sets of plans is shown in Table 1, and the data in Table 2 are records randomly extracted from traffic information.

\subsection{Optimization of Traffic Signals Based on Microbial Genetic Algorithm}

2.3.1. Experiment Procedure. Genetic algorithm is a global random search algorithm that simulates the evolutionary process and natural selection process of natural species. The algorithm was proposed by Professor J. Holland of Michigan University in 1975. This method was based on genetic theory and natural selection process [21] and used the natural selection rule and the cross mutation process of chromosomes and genes in biological groups as simulation object [22-24]. In this paper, the optimization process of microbial genetic algorithm is as follows:

(1) Initialize the values of 4 decision variables randomly (effective green light time for 4 phases).

(2) Binary coding is used to encode the individual values in this population to generate an initial population of about 300 .

(3) The target value is $\left(\alpha D / D_{0}\right)-\left(\beta B / B_{0}\right)$; we hope the expected target value in this article is as small as possible; the ultimate goal is to get a value, so when the objective function can take the minimum value, what are the values of the corresponding 4 decision variables? 


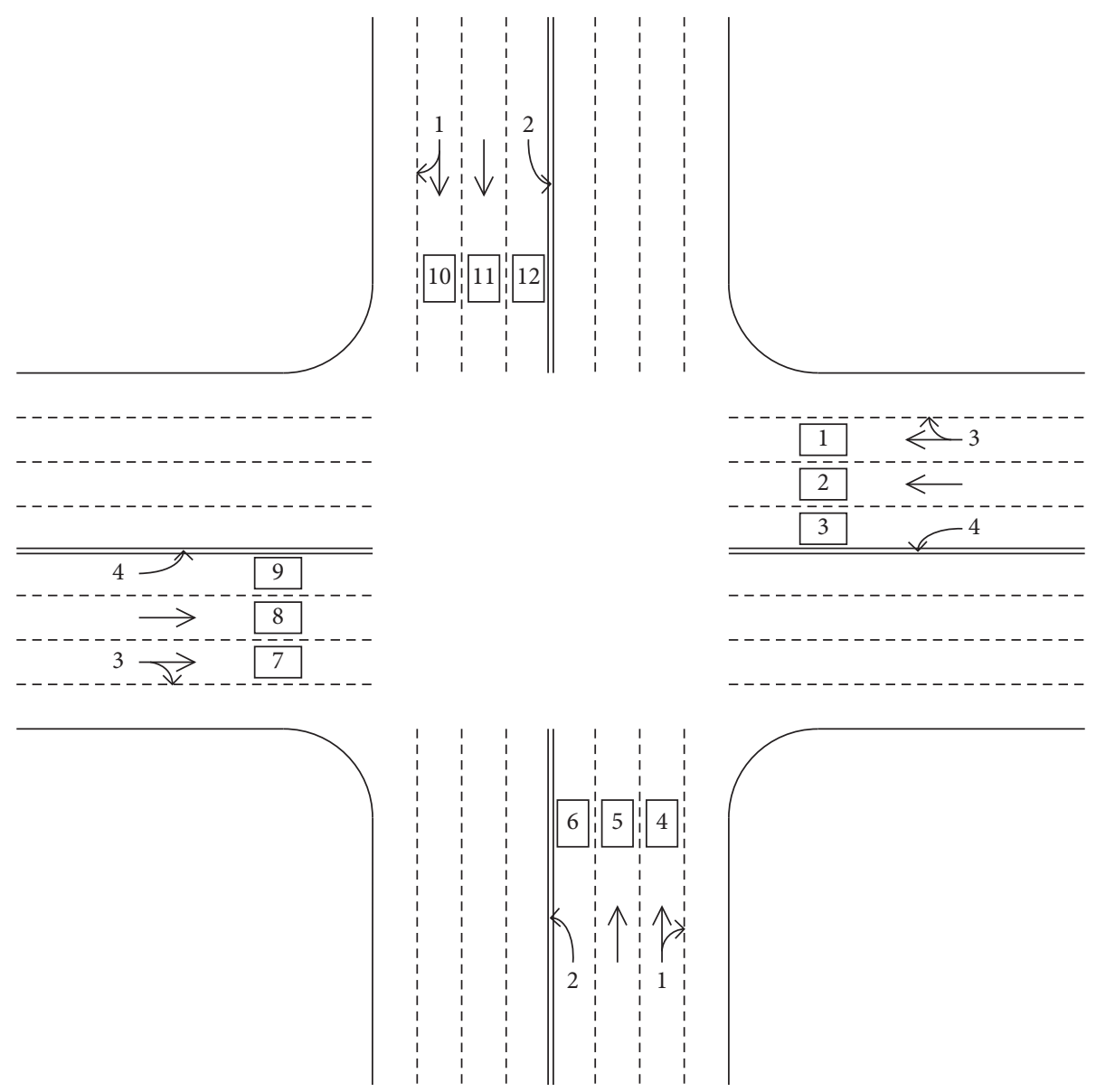

FIgURE 2: City intersection simulation map.

TABLE 1: Timing schemes for the SCATS.

\begin{tabular}{lcccc}
\hline $\begin{array}{l}\text { Timing } \\
\text { scheme }\end{array}$ & $\begin{array}{c}\text { Phase } A \text { green light duration } \\
(\%)\end{array}$ & $\begin{array}{c}\text { Phase } B \text { green light duration } \\
(\%)\end{array}$ & $\begin{array}{c}\text { Phase } C \text { green light duration } \\
(\%)\end{array}$ & $\begin{array}{c}\text { Phase } D \text { green light duration } \\
(\%)\end{array}$ \\
\hline 1 & 29 & 24 & 24 & 23 \\
2 & 35 & 19 & 28 & 18 \\
3 & 24 & 24 & 26 & 26 \\
4 & 25 & 24 & 27 & 24 \\
\hline
\end{tabular}

(4) Two individuals are randomly selected from the population pop, and the two individuals are calculated to obtain the objective function value; according to the objective value, the fitness of the two individuals can be calculated.

(5) According to their fitness, these two individuals are defined as winner and loser.

(6) For the winner, make sure it does not change; for the loser, make it a crossover; the object of the cross comes from the winner; put the loser back in the original pop.

(7) Through crossover, the loser has a certain chance to perform mutation operations, determine the position of chromosomal mutations, and perform corresponding mutations according to different coding strategies; then, a new generation of groups has emerged.
(8) Repeat steps (3)-(7) and update the 4 decision variables from generation to generation until the number of iterations reaches the original preset value.

2.3.2. Experimental Results. Time dependent objective function is shown in Figure 3.

In the figure, the abscissa represents the number of iterations, and the ordinate represents the value of the objective function at the end. The figure visualizes the entire optimization process of the microbial genetic algorithm. It is clear from the figure that the value of the corresponding objective function changes with the increase of iteration [25]. Before the objective function completes 24 iterations, the value of the objective function fluctuates with the accumulation of the iteration and the displayed state is unstable, but the value of the objective function tends to be stable, approaching 25 iterations [26]. Therefore, it is not 
TABLE 2: Information statistics of traffic flow.

\begin{tabular}{lccc}
\hline Phase & Coil number & Green light duration & Number of vehicles \\
\hline$C$ & 1 & 36 & 6 \\
$C$ & 2 & 36 & 7 \\
$D$ & 3 & 24 & 3 \\
$C$ & 7 & 36 & 2 \\
$C$ & 8 & 36 & 2 \\
$D$ & 9 & 24 & 2 \\
$A$ & 4 & 44 & 0 \\
$A$ & 5 & 44 & 0 \\
$A$ & 10 & 44 & 4 \\
$A$ & 11 & 44 & 7 \\
$C$ & 1 & 32 & 3 \\
$C$ & 2 & 32 & 3 \\
$B$ & 6 & 26 & 0 \\
$C$ & 7 & 32 & 0 \\
$C$ & 8 & 32 & 2 \\
$B$ & 12 & 26 & 0 \\
$A$ & 4 & 51 & 0 \\
$A$ & 5 & 51 & 0 \\
$A$ & 10 & 51 & 6 \\
$A$ & 11 & 51 & 10 \\
$\vdots$ & $\vdots$ & $\vdots$ & $\vdots$ \\
\hline
\end{tabular}

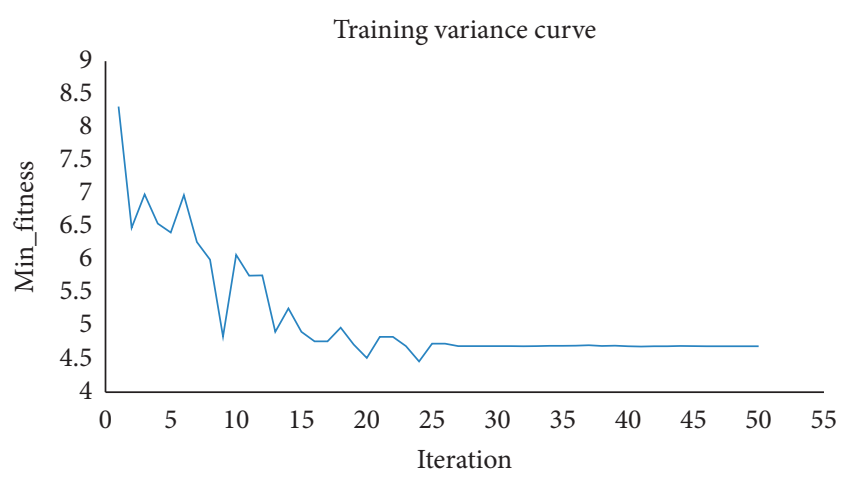

FIGURE 3: Objective function changes with the increase of iteration.

difficult to conclude from the relationship change diagram that, in terms of accuracy and training time, the microbial genetic algorithm performed better.

The traffic data is derived from the traffic condition information of an-hour-long evening rush in a certain domestic city. Recording the values of vehicle delay $D$, traffic capacity $B$, and objective function PI during this period and comparing them with the SCATS at the same time, we get the results in Table 3.

As the data in Table 3 show, we set the same external environmental factors, taking the same vehicle information in the same time period for comparison. The microbial genetic algorithm has improved by $67.465 \%$ in optimizing the objective function [27]. Obviously, the microbial genetic algorithm proposed in this paper is superior to SCATS in traffic signal optimization.

The SCATS is the basis of the algorithm optimization in this paper, with a period of $230 \mathrm{~s}$. The time given in Table 4 is the effective green light time when the microbial genetic algorithm obtains the best solution.
TABLE 3: Comparison of performance parameters between SCATS and MGA.

\begin{tabular}{lccc}
\hline & Vehicle delay $(\mathrm{s})$ & Capacity $(\mathrm{v} / \mathrm{h})$ & PI \\
\hline SCATS & 4191.56723 & 3999.30131 & 14.56855 \\
MGA & 1430.77263 & 3189.67742 & 4.73989 \\
\hline
\end{tabular}

TABLE 4: Green light duration of each phase obtained by GMA.

\begin{tabular}{lc}
\hline Phase number & Green light duration $(\mathrm{s})$ \\
\hline$A$ & 15.00 \\
$B$ & 33.00 \\
$C$ & 15.00 \\
$D$ & 10.00 \\
\hline
\end{tabular}

\subsection{Signal Optimization Based on Simulated Annealing Algorithm}

2.4.1. Algorithm Research History and Main Announcement. American physicist N. Metropolis and colleagues published an article in 1953 that studied complex systems and calculated the energy distribution in them. They used Monte Carlo simulation to calculate the energy distribution of molecules in multimolecular systems [28]. This is a term often referred to in the simulated annealing algorithm as the Metropolis criterion.

In 1983, physicists S. Kirkpatrick, C. D. Gelatt, and M. P. Vecchi of the IBM Corporation in the United States borrowed the method of Metropolis and others to explore a rotating glass state system [29]; they discovered the energy of the physical system and the cost of some optimal combination problems. The function is quite similar: seeking the lowest cost is like seeking the lowest energy. As a result, they developed an algorithm based on the metropolitan process to find the best solution. The optimal solution (the maximum value) of the problem is similar to the minimum energy of the system; when the system cools down, the energy also gradually decreases, and in the same sense, the solution to the problem falls down to its maximum. The calculation formula for the Metropolis criterion is as follows:

$$
p= \begin{cases}1, & \text { if } E\left(x_{\text {new }}\right)<E\left(x_{\text {old }}\right), \\ \exp \left(-\frac{E\left(x_{\text {new }}\right)-E\left(x_{\text {old }}\right)}{\mathrm{T}}\right), & \text { if } E\left(x_{\text {new }}\right) \geq E\left(x_{\text {old }}\right),\end{cases}
$$

$$
p(d E)=\exp \left(\frac{d E}{K T}\right)
$$

In (11) and (12), $p$ is the probability of cooling, $K$ is the constant, and exp is the natural index.

The cooling calculation formula of the simulated annealing algorithm is as follows:

$$
T(t)=\frac{T_{0}}{1+t} .
$$




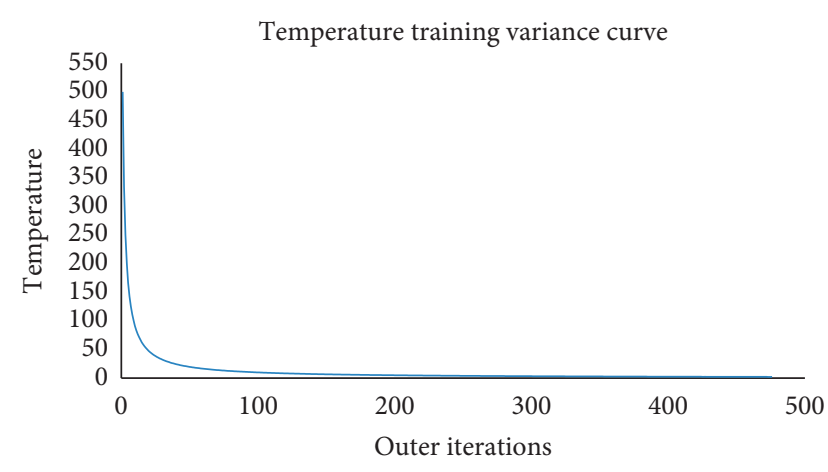

Figure 4: Relationship between the temperature and the number of outer iterations.

In (13), $T_{0}$ is the initial temperature.

In the process, the maximum tempering temperature gradually decreases with each annealing process, and the algorithm operation is stopped until the tempering end temperature is reached. The tempering process during the inner loop of the algorithm increases the algorithm's ability to accept nonoptimal solutions, which helps the algorithm to jump out of the local optimal solution and obtain the global optimal solution.

2.4.2. Experimental Results. In this simulation algorithm, the relationship between the temperature and the iterative algorithm in the driving process, and between the objective function and the external iterative algorithm is as follows.

Figure 4 shows the linear change in temperature as the number of iterations increases. Meanwhile, in another aspect, what is presented in Figure 5 is the change corresponding to the objective function when the number of iterations is accumulated. During the accumulation of the number of iterations, the temperature decreases accordingly. When the temperature reaches $0.4^{\circ} \mathrm{C}$, the functional relationship is stable and convergent. In the graph of the relationship between the objective function and the number of outer iterations, the abscissa is each outer loop, and the ordinate is the objective function value. It can be seen that with the decrease of iteration depth and temperature, the value of the objective function gradually becomes smaller [30]. The optimization effect of the simulated annealing algorithm is getting better and better. Meanwhile, it can be seen from the figure that, at the 500th cycle, the SA model has approached convergence and the target value is around 7.7.

Similar to the microbial genetic algorithm, the values of vehicle delay $D$, traffic capacity $B$, and objective function PI are also recorded. The recorded data is shown in Table 5 . Compared with the SCATS timing scheme, the objective function is improved by $46.848 \%$.

By setting the same variables and taking the same dataset, the simulated annealing algorithm has been partially improved compared to the timing of the SCATS. The time given in Table 6 is the effective green light time when the simulated annealing algorithm obtains the best solution.

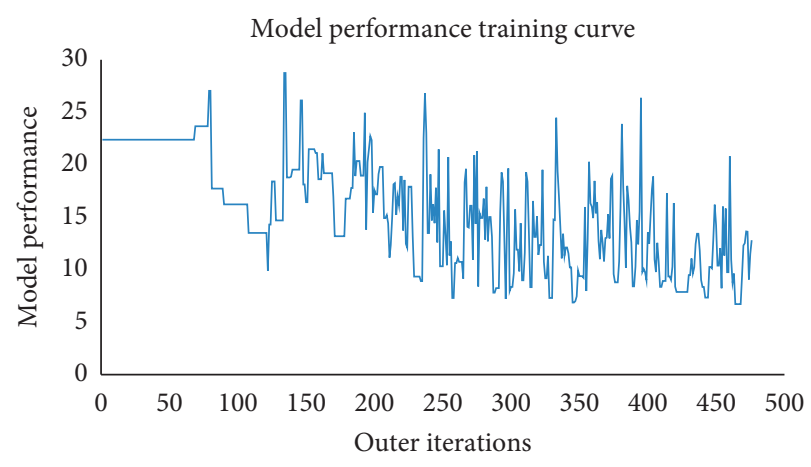

FIgURE 5: Relationship between the objective function and the number of outer iterations.

TABLE 5: Comparison of performance parameters between SCATS and SA.

\begin{tabular}{lccc}
\hline & Vehicle delay $(\mathrm{s})$ & Capacity $(\mathrm{v} / \mathrm{h})$ & PI \\
\hline SCATS & 4191.56723 & 3999.30131 & 14.56855 \\
SA & 2286.56378 & 3778.47053 & 7.74342 \\
\hline
\end{tabular}

TABLE 6: Green light duration of each phase obtained by SA.

\begin{tabular}{lc}
\hline Phase number & Green light duration $(\mathrm{s})$ \\
\hline$A$ & 28.47 \\
$B$ & 25.43 \\
$C$ & 29.13 \\
$D$ & 17.49 \\
\hline
\end{tabular}

\section{Conclusions}

This paper uses two algorithms to optimize the signal timing scheme of intersections. The innovation of this paper lies in the use of mutation genetic algorithm and simulated annealing algorithm to optimize the timing scheme of SCATS. Through the real traffic flow data, the effectiveness of the two optimization algorithms in improving the objective function is demonstrated.

The advantage of the microbial genetic algorithm is that it will select the one with poor fitness from the two individuals as the mutation object; therefore, the quality of the optimal solution can be improved. At the same time, the microbial genetic algorithm is not highly dependent on the initial population, and two individuals can be randomly selected to calculate their objective function value and individual fitness. The global search ability of the algorithm is robust, and it will not fall into the trap of local optimal solution.

The advantage of the simulated annealing algorithm is that its entire operation process is very simple. The algorithm has strong robustness and applicability. Simultaneously, in order to ensure that the global optimal solution is not missed during the solution process, a higher initial temperature is set.

Both algorithms require a lot of iterations, so there are drawbacks in solving real-time problems. On the one hand, the signal optimization in this paper is only for a specific 
intersection, which lacks general applicability. On the other hand, when selecting the objective function, other traffic factors are not fully considered, and there are certain errors here. Future research on signal control of intersections may consider more parameters, and the vehicle condition information of adjacent intersections will also affect that of the others. Incorporating more variables into the reference model, extending the research on individual intersections to intersection groups, and comparing the original SCATS timing scheme with actual traffic flow data can be the directions of hard research in the future.

\section{Data Availability}

The data used to support the findings of this study are included within the article.

\section{Conflicts of Interest}

The authors declare no conflicts of interest regarding the publication of this paper.

\section{Acknowledgments}

This work was supported by School of Information and Communication, Guilin University of Electronics Technology, "Research on Energy Efficiency Evaluation Mechanism of Next Generation Wireless Communication Service Transmission for Industry 4.0" (project number: NF150261), "High Precision Time-Frequency Transmission and Comparative Study" (project number: NF19002X) and CERNET Innovation Project (NGII20160410).

\section{References}

[1] L. I. U. Quan-Fu, B.-C. Lu, M. A. Qing-Lu, and J. Deng, "Traffic signal control multi-objective optimization at intersection," Technology \& Economy in Areas of Communications, vol. 16, no. 1, pp. 47-50, 2014.

[2] Y.-F. Gao, H. Hu, H. Han, and X.-G. Yang, "Multi-objective optimization and simulation for urban road intersection group traffic signal control," China Journal of Highway and Transport, vol. 25, no. 6, pp. 129-135, 2012.

[3] A. Flannery, K. Wochinger, and A. Martin, "Driver assessment of service quality on urban streets," Transportation Research Record: Journal of the Transportation Research Board, vol. 1920, no. 1, pp. 25-31, 2005.

[4] F. V. Webster, Traffic Signal Settings, Road Research Laboratory, London, UK, 1958.

[5] B. G. Heydecker, "A decomposition approach for signal optimisation in road networks," Transportation Research Part B: Methodological, vol. 30, no. 2, pp. 99-114, 1996.

[6] W.-M. Wey, "Model formulation and solution algorithm of traffic signal control in an urban network," Computers, Environment and Urban Systems, vol. 24, no. 4, pp. 355-378, 2000.

[7] D. I. Robertson, TRANSYT: A Traffic Network Study Tool, Road Research Laboratory, Crowthorne, UK, 1969.

[8] S. C. Wong, W. T. Wong, C. M. Leung, and C. O. Tong, "Group-based optimization of a time-dependent TRANSYT traffic model for area traffic control," Transportation Research Part B: Methodological, vol. 36, no. 4, pp. 291-312, 2002.
[9] J.-Q. Zeng, J.-J. Wang, K. Liu et al., "CTMMOGA based crossroad traffic signal control," Journal of University of Science and Technology of China, vol. 35, no. 2, pp. 284-290, 2005.

[10] X.-S. Yao, X. Luo, and D. U. Jin-You, “Traffic signal control based on satisfactory multi-optimization," Computer Engineering and Applications, vol. 36, no. 35, pp. 9-10, 2006.

[11] Y.-Y. Ma, X.-G. Yang, and Y. Zeng, "Multi objective cycle length optimization model and solution," Journal of Tongji University: Natural Science, vol. 37, no. 6, pp. 761-765, 2009.

[12] R.-M. Li and H.-P. Lu, "Traffic signal control multi-object optimization based on genetic algorithm," Journal of Chang'an University: Natural Science Edition, vol. 29, no. 3, pp. 85-88, 2009.

[13] C.-T. Cao and J.-M. Xu, "Multi-object traffic signal control method for single intersection," Computer Engineering and Applications, vol. 46, no. 16, pp. 20-22, 2010.

[14] K.-H. Gao, Reach on Bus Priority Signal Coordination Control and Simulation in Urban Road Intersections, Beijing Jiaotong University, Beijing, China, 2010.

[15] G. P. Liu, J. F. Whidborne, and J. B. Yang, Multi-objective Optimisation and Control, Research Studies Press Ltd., London, UK, 2003.

[16] L. F. C. Gonzalez, Discharge Headway at Signalized Intersections in Monterrey, Doetoral Dissertation of University of Texas, USA, Nuevo Leon, Mexico, 2006.

[17] M. Gentili and P. B. Mirchandani, "Locating sensors on traffic networks: models, challenges and research opportunities," Transportation Research Part C: Emerging Technologies, vol. 24, pp. 227-255, 2012.

[18] A. Stevanovic, Adaptive Traffic Control Systems: Domestic and Foreign State of Practice, Transportation Research Board, Washington, DC, USA, 2010.

[19] F. V. Webster, "Traffic signal settings," Department of Scientific and Industrial Research, London, UK, Road Research Technical Paper No 39, 1958.

[20] J. Lu, S. Dissanayake, and N. Castillo, Safety Evaluation of Right Turns Followed by U-Turns as an Alternative to Direct Left Turns Conflict Analysis, Research Report, Department of Civil \& Environmental Engineering, University of South Florida Institution, Tampa, FL, USA, 2001.

[21] H. Shen, "A study of welding robot path planning application based on genetic ant colony hybrid algorithm," in Proceedings of the 2016 IEEE Advanced Information Management, Communicates, Electronic and Automation Control Conference (IMCEC), Xi'an, China, October 2016.

[22] O. Kramer, Genetic Algorithm Essentials. Studies in Computational Intelligence, Springer, Berlin, Germany, 2017.

[23] D. Hermawanto, "Genetic algorithm for solving simple mathematical equality problem," Computer Science, 2017.

[24] X. Li and L. Gao, "An effective hybrid genetic algorithm and tabu search for flexible job shop scheduling problem," International Journal of Production Economics, vol. 174, pp. 93-110, 2016.

[25] Y. Hou, "Research and implementation of hybrid clustering algorithm in big data processing," in Proceedings of the 2018 International Conference on Transportation \& Logistics, Information \& Communication, Smart City (TLICSC 2018), Chengdu, China, December 2018.

[26] Y. Meng, J. Zhang, and J. Cai, "Improved binary decision diagram based accelerated circuit evolutionary algorithm," in Proceedings of the 2015 Joint International Mechanical, Electronic and Information Technology Conference, Chongqing, China, December 2015. 
[27] Q. Sun, T. Zhou, and H. Li, "A novel register allocation algorithm for testability," Tsinghua Science \& Technology, vol. 12, no. S1, pp. 57-60, 2007.

[28] J. Tang, C. Deng, and G.-B. Huang, "Extreme learning machine for multilayer perceptron," IEEE Transactions on Neural Networks and Learning Systems, vol. 27, no. 4, pp. 809-821, 2016.

[29] M. M. Mafarja and S. Mirjalili, "Hybrid whale optimization algorithm with simulated annealing for feature selection," Neurocomputing, vol. 260, pp. 302-312, 2017.

[30] Z.-Q. Wang, N.-J. Zhou, J.-Q. Zhang, J. Guo, and X.-Y. Wang, "Parametric optimization and performance comparison of organic Rankine cycle with simulated annealing algorithm," Journal of Central South University, vol. 19, no. 9, pp. 25842590, 2012. 